\title{
Interaction of anthracene and its oxidative derivatives with human serum albumin $\star$
}

\author{
Katarzyna Skupińska ${ }^{1}$, Monika Zylm¹ ${ }^{1}$ Irena Misiewicz ${ }^{1}$ and \\ Teresa Kasprzycka-Guttman ${ }^{1,2 \bowtie}$ \\ ${ }^{1}$ Confocal Microscopy Laboratory, National Institute of Public Health, Warszawa Poland; ${ }^{2}$ Department of Chem- \\ istry, Warsaw University, Warszawa, Poland; ${ }_{e}$-mail: guttman@il.waw.pl
}

Received: 12 April, 2005; revised: 24 October, 2005; accepted: 16 December, 2005

available on-line: 09 January, 2006

\begin{abstract}
Binding affinities of ten polycyclic aromatic hydrocarbons to albumin were determined: anthracene, its eight oxy-derivatives: anthraquinone, 9-anthracenemethanol, 9-anthraldehyde, 9-anthracenecarboxylic acid, 1,4-dihydroxyanthraquinone, 1,5-dihydroxyanthraquinone, 1,8-dihydroxyanthraquinone, 2,6-dihydroxyanthraquinone and benzo[a]pyrene. The quenching of albumin fluorescence was used to measure the $\mathrm{PAH}$ - protein interaction. The theoretical curve of calculated fluorescence was fitted to experimental data after necessary corrections regarding PAHs fluorescence and inner filter effect. From the numerical fitting the final association constants were calculated. Anthracene and anthraquinone failed to quench the albumin fluorescence. 9-anthracenecarboxylic acid showed the highest, while 9-anthracenemethanol the weakest albumin binding affinity. The affinity constants determined for 9-anthraldehyde and benzo[a]pyrene were of the same magnitude and indicated low-affinity binding to albumin. The constants obtained for the four dihydroxyanthraquinones were higher, but dissimilar, which suggests that the position of the functional group in anthracene molecule influences the binding constant. Moreover, this study suggests that the type of substituent plays a significant role in PAH-albumin complex formation. The carboxylic group increases the binding affinity of the anthracene molecule the most rather than the presence of both carbonyl and hydroxyl groups. The lowest affinity constants were obtained for aldehyde, methyl and carbonyl substituents.
\end{abstract}

Keywords: polycyclic aromatic hydrocarbons, albumin, affinity constant

Anthracene is a three-ring compound belonging to polycyclic aromatic hydrocarbons (PAHs). PAHs are environmental pollutants and some of them are considered carcinogenic to humans. At least several PAHs have been shown to induce tumors in experimental animals (Wang et al., 1995; Pashko \& Schwartz, 1996; Kumar et al., 2001; Flesher et al., 2002). Epidemiological studies on humans revealed also more frequent incidence of skin cancer in chimney sweepers exposed to coal tar. People are exposed to PAHs mainly at the workplace, however, many PAHs are found in tobacco smoke or diet (Philips, 1999).

The PAHs present in the environment are considered as non active and unable to cause car- cinogenesis directly. To become reactive, they must be firstly metabolized. PAHs are metabolized by many enzymes, primarily by cytochrome P450 enzymes: CYP1A1, CYP1A2 and CYP1B1 and by epoxide hydrolase. As products of these reactions many oxy-derivatives can be obtained, e.g.: epoxides, dihydrodiols, diol-epoxides, phenols and quinones (Prodi et al., 1984; Lecoq et al., 1991; Otto et al., 1992; Honey et al., 2000; Willett et al., 2000; Hecht et al., 2003; Lee et al., 2003; Pangrekar et al., 2003). The diolepoxides have been shown to bind to biological molecules like DNA or proteins. Such PAH derivatives-DNA adducts can cause mutations and in consequence carcinogenesis and tumor development (Garner, 1998; Boysen \& Hecht, 2003).

$\star$ Preliminary report on this subject was presented at the 8th International Congress on Amino Acids and Proteins. September, 2003 Roma, Italy.

Abbreviations: HSA, human serum albumin; PAH(s), polycyclic aromatic hydrocarbon(s). 
PAHs adducts with biomolecules are used as biomarkers in order to assess human exposure to PAHs. A number of studies have been conducted to evaluate the usability of these biomarkers (Nielsen et al., 1996; Sram \& Binkova, 2000; Jacob \& Seidel, 2002; Ruchirawat et al., 2002). PAH-albumin adducts were found to be very useful biomarkers since albumin is the most abundant protein in the blood. Moreover, no repair occurs in protein so the adduct level is a total sum of human exposure to PAHs which took place during the lifetime of the protein. It was shown also that the level of PAH-albumin adducts depends on the duration of exposure (Lee et al., 1991; Stella et al., 1995; Ehrenberg et al., 1996).

Albumin is a predominantly $\alpha$-helical protein consisting of 585 amino acids and is built of three domains. This protein has an ability to bind covalently or reversibly a number of various endo- and exogenous compounds (Kragh-Hansen, 1990). Up to now various classes of binding sites have been determined. It was shown that albumin has one specific binding site for PAHs - either histidine-146 or lysine-137 located in subdomain IB near the single tryptophan-214 which is a fluorescent residue of albumin. Lysine-385 located in subdomain IIIA was also proposed to be a binding site (Tannenbaum et al., 1993; Brunmark et al., 1997). Fabriciova et al. (2004) showed that the binding sites for dihydroxyanthraquinones are localized in subdomains IIA and IIIA of albumin. These two subdomains are believed to be a general binding site for small organic molecules. These subdomains are also characteristic for binding drugs like warfarin (IIA), ibuprofen (IIIA) or oxazepan (IIIA) which consist of benzene rings (Epps et al., 1995).

It has been shown that energy transfer is possible from the single tryptophan residue to the PAHs bound, which can result in albumin fluorescence quenching (Ma et al., 1977). Albumin fluorescence quenching as a result of ligand binding was also described for albumin interactions with drugs and proteins (Epps et al., 1995; Gou et al., 2004; Qg et al., 2005). In our work this phenomenon was applied to determine the binding constants of albumin and PAHs and their oxo-derivatives. Ten different compounds of these groups were selected, varying in the aromatic ring number and structure as well as in the type of oxy-substituents (carbonyl, carboxyl and hydroxyl) and their position in the anthracene molecule chosen by us as a representative. Our results are the first approach in calculations of anthracene derivatives-albumin dissociation constant as, according to our knowledge, there is no literature data on the affinity of these compounds toward albumin. The aim of this study was to establish if PAHs unsubstituted and substituted by oxygen-containing substituents can bind to albumin, and how the type, number and position of the substituents influence the binding affinity.

\section{MATERIALS AND METHODS}

Chemicals. All PAHs derivatives (abbreviated further as $\mathrm{PAH}$ ): anthracene, anthraquinone, 9anthracenemethanol, 9-anthraldehyde, 9-anthracenecarboxylic acid, 1,4-dihydroxyanthraquinone, 1,5dihydroxyanthraquinone, 1,8-dihydroxyanthraquinone, 2,6-dihydroxyanthraquinone, benzo[a]pyrene and human serum albumin (Fraction V) were purchased from Sigma Chemical Co. The purity of all compounds were in the range 99.6-99.9\%. All PAHs were dissolved in ethanol and albumin was dissolved in phosphate buffered saline (PBS) $(0.05 \mathrm{M}$, $\mathrm{pH}$ 7.4). The solvents were purchased from POCh (Poland).

Spectroscopic measurements. Absorbance measurement was carried out with a UV-VIS Shimadzu Uvmini1240 spectrometer in a $1 \times 1 \mathrm{~cm} \mathrm{cu}-$ vette.

A Shimadzu RF-5000 spectrofluorimeter was used to measure the fluorescence of albumin-PAH solutions. The mixture in a $1 \times 1 \mathrm{~cm}$ quartz cuvette was stirred continuously during measurement and the temperature was kept constant at $25^{\circ} \mathrm{C}$. The albumin fluorescence was measured at the excitation wavelength of $278 \mathrm{~nm}$ and the signal was observed at $340 \mathrm{~nm}$ (the excitation slit width was set at $3 \mathrm{~nm}$ and emission slit width at $10 \mathrm{~nm}$ ). These conditions were chosen to ensure strongest albumin fluorescence signal.

Albumin stock solutions in PBS were prepared prior to experiment and the final concentration of $0.5 \mu \mathrm{M}$ was confirmed by absorbance measurement $\left(\varepsilon_{278}=37000 \mathrm{M}^{-1} \mathrm{~cm}^{-1}\right)$. This concentration was chosen as addition of increasing amounts of PAHs resulted in a fluorescence decrease down to a minimal value. The albumin solution was prewarmed to $25^{\circ} \mathrm{C}$ in the cuvette for $15 \mathrm{~min}$ and then fluorescence was measured until stabilized. PAHs were dissolved in ethanol until saturation and several dilutions of such solutions were prepared. Their concentrations were checked by means of spectroscopic measurements. Titration with PAHs was performed by adding aliquots of PAHs solutions to $2900 \mu \mathrm{L}$ of albumin dissolved in PBS so as to achieve uniformly increasing PAHs concentrations in the cuvette. The final PAHs concentrations were as follows: anthracene 0.003-2.793 $\mu \mathrm{M}$, anthraquinone 0.005-7.07 $\mu \mathrm{M}$, 9-anthracenemethanol 0.002-44.665 $\mu \mathrm{M}, 9$-anthraldehyde 0.002-25.402 $\mu \mathrm{M}, 9$-anthracenecarboxylic acid 0.034-8.583 $\mu \mathrm{M}, 1$,4-dihydroxyanthraquinone 0.0003$4.971 \mu \mathrm{M}, 1,5$-dihydroxyanthraquinone $0.003-1.873$ $\mu \mathrm{M}, 1,8$-dihydroxyanthraquinone $0.03-25.248 \mu \mathrm{M}$, 
2,6-dihydroxyanthraquinone $0.002-7.638 \mu \mathrm{M}$ and benzo[a]pyrene $0.002-25.193 \mu \mathrm{M}$. After addition of each PAH sample the fluorescence was measured according to literature data (Niedzwiecka et al., 2002; Zuberek et al., 2003): fluorescence signal was registered 4 times during $40 \mathrm{~s}$ and mean \pm S.D. was calculated. Then, there was a $20 \mathrm{~s}$ break for the next sample addition. Each titration was repeated independently 5 times. The total amount of PAHs in ethanol never exceeded $100 \mu \mathrm{l}(3.3 \%$, v/v). Such concentration of ethanol was shown not to disturb albumin fluorescence.

Additional titrations were performed to determine the fluorescence of increasing amounts of ethanol in PBS and PBS was titrated with PAHs to verify if the fluorescence of any PAH occurs. All these measurements were performed under the same conditions (including the volume of aliquots added) as the albumin titrations. The absorbance of increasing concentrations of PAHs was also determined for the excitation and emission wavelength of albumin in order to calculate the inner filter effect.

Data analysis. In order to determine the dissociation constants, two methods are usually applied: the protein concentration is kept constant while ligand concentration is increased from a concentration lower than that of albumin to a higher one. Another option is to keep the ligand concentration constant and to increase the protein concentration, however, this one has not been well studied yet (Epps et al., 1995; 1999). In this study PAHs were added to a constant concentration of albumin and the fluorescence of the solutions was measured.

Binding of PAHs to $\mathrm{n}$ identical sites in albumin can be described by the Scatchard equation:

$\mathrm{n}=\frac{n L}{K+L}$

where

$\mathrm{v}$ - average number of ligand molecules bound per protein molecule,

$\mathrm{n}$ - number of binding sites,

$\mathrm{L}$ - free (unbound) ligand concentration,

$K$ - dissociation constant of ligand-albumin complex.

However, v can be written by an other equation:

$\mathrm{n}=\frac{L_{0}-L}{A_{0}}$

where

$\mathrm{L}_{0}$ - total ligand concentration,

$\mathrm{A}_{0}$ - total albumin concentration.

The fluorescence of albumin is quenched by

ligand binding and is the sum of free albumin and albumin-ligand complex fluorescences:

$\mathrm{F}=\mathrm{f}_{\mathrm{A}}\left(\mathrm{A}_{0}-\mathrm{AL}\right)+\mathrm{f}_{\mathrm{AL}} \mathrm{AL}=\mathrm{F}_{0}-\left(\mathrm{f}_{\mathrm{A}}-\mathrm{f}_{\mathrm{AL}}\right) \mathrm{AL}$ where

$\mathrm{AL}$ - concentration of complexed albumin,

$f_{A}$ and $f_{A L}$ are molar emissivity of free and complexed albumin.

The AL concentration can be calculated from the formula:

$A L=\frac{L_{0}-L}{n}$

It follows from calculations (Eqns. 1, 2 and 4) arises that the complex concentration (AL) is described by the following equation:

$A L=\frac{\left(n \cdot A_{0}+L_{0}+K\right)-\sqrt{\left(n \cdot A_{0}+L_{o}+K\right)^{2}-4 \cdot n \cdot A_{0} \cdot L_{o}}}{2 \cdot n}$

Hence, substituting the calculated value of AL (Eqn. 5) to Eqn. 3, the fluorescence of the mixture can be expressed as (Guo et al., 2004; Zuberek et al., 2003):

$F=F_{o}-\left(f_{A}-f_{A L}\right) \cdot \frac{\left(n \cdot A_{o}+L_{o}+K\right)-\sqrt{\left(n \cdot A_{o}+L_{o}+K\right)^{2}-4 \cdot n \cdot A_{o} \cdot L_{o}}}{2 \cdot n}$

This equation yields information about the dissociation constant and also about the binding stoichiometry.

The above equation is correct only if the binding sites specific for PAHs in albumin are identical to each other and act independently. Moreover, this formula is correct only for strong binding sites. In the case of low affinity ligands, a modified equation describes the fluorescence of the mixture (the case of ligand excess with respect to albumin). Unfortunately this method does not supply information on the stoichiometry of the binding. However, when fitting Eqn. 6 gives no results, a low affinity of a compound toward albumin is to be assumed and the following equation is fitted:

$\mathrm{F}=\mathrm{F}_{\mathrm{o}}-\left(\mathrm{f}_{\mathrm{A}}-\mathrm{F}_{\mathrm{AL}}\right) \frac{\mathrm{A}_{0} \cdot \mathrm{L}_{0}}{\mathrm{~K}+\mathrm{L}_{0}}$

Using the above equations the experimental dependence of $\mathrm{F}$ on $\mathrm{L}_{\mathrm{o}}$ was analyzed by non-linear least-squares regression. From the best fit, the $K$ value was calculated. The fittings were performed with SigmaPlot 2000 SPSS Inc.

\section{Corrections}

PAHs fluorescence. Some of the compounds studied exhibit fluorescence in the excitation/emission wavelengths specific for albumin. In this case the fluorescence of the mixture consists of free albumin fluorescence, fluorescence of the complex and fluorescence of PAH. After an initial fluorescence drop, a total fluorescence signal increase can be observed for high concentration of PAHs as a result of 
predominant ligand fluorescence. Then corrections must be done. The modified equations describing solution fluorescence in dependence on ligand concentration will be applied:

$$
\begin{aligned}
& \mathrm{F}=\mathrm{F}_{\mathrm{o}}+\mathrm{f}_{\mathrm{L}} \cdot \mathrm{L}_{\circ}-f \cdot \frac{\left(\mathrm{n} \cdot \mathrm{A}_{\odot}+\mathrm{L}_{\odot}+\mathrm{K}\right)-\sqrt{\left(\mathrm{n} \cdot \mathrm{A}_{\odot}+\mathrm{L}_{\circ}+\mathrm{K}\right)^{2}-4 \cdot \mathrm{n} \cdot \mathrm{A}_{\odot} \cdot \mathrm{L}_{\odot}}}{2 \cdot \mathrm{n}} \\
& \mathrm{f}=\mathrm{f}_{\mathrm{L}}+\mathrm{f}_{\mathrm{A}}-\mathrm{f}_{\mathrm{AL}}
\end{aligned}
$$

where $f_{L}-$ molar emissivity of free (unbound) ligand, $f_{A}-$ molar emissivity of free albumin, and $f_{A L}$ - molar emissivity of albumin-PAH complex. For low-affinity ligands:

$$
\mathrm{F}=\mathrm{F}_{\mathrm{o}}+f_{L} \cdot L_{o}-f \cdot \mathrm{A}_{\mathrm{o}} \frac{\mathrm{L}_{0}}{\mathrm{~K}+\mathrm{L}_{\mathrm{o}}}
$$

Inner filter effect. The absorbance spectra of all compounds studied overlap the excitation and emission wavelengths of albumin. Therefore, the compounds absorb the light used to excite albumin as well as the light emitted by albumin. All data were corrected and the experimental fluorescence values were multiplied by factor $10 \frac{\left(\varepsilon_{\mathrm{exc}}+\varepsilon_{\mathrm{em}}\right) L_{0} l}{2}$ where $\varepsilon_{\text {exc }}$ - ligand molar extinction coefficient at the albumin excitation wavelength, $\varepsilon_{\mathrm{em}}$ - ligand molar extinction coefficient at the albumin emission wavelength, $\mathrm{L}_{\mathrm{o}}$ - ligand concentration, 1 - optical length. The coefficients were determined before the main experiments by fitting a line to experimentally determined absorbance values for increasing concentrations of PAHs.

Other corrections. Other corrections include the albumin dilution during titration and the background fluorescence of PBS and ethanol, which were subtracted from experimental values.

\section{RESULTS}

Ten PAHs were studied regarding the influence of structure differences on HSA affinity. These PAHs were: anthracene and its eight oxy-derivatives: anthraquinone, 9-anthracenemethanol, 9-anthraldehyde, 9-anthracenecarboxylic acid, 1,4-dihydroxyanthraquinone, 1,5-dihydroxyanthraquinone, 1,8-dihydroxyanthraquinone, 2,6-dihydroxyanthraquinone as well as benzo[a]pyrene, which is considered carcinogenic to people. Anthracene is not recognized as a carcinogen, however, it is toxic toward people and hence specified by US EPA as a hazardous substance. Quenching of albumin intrinsic fluorescence was the method used in order to determine the dissociation constant of the PAH - albumin complexes. In the study fraction $\mathrm{V}$ human albumin was used in order to mimic the conditions of human blood plasma.

All PAHs were dissolved to achieve the highest concentrations possible and series of dilutions were prepared. Aliquots of these PAHs dilutions were added to albumin solution in order to achieve increasing concentrations of PAH in the measured samples and the mixture fluorescence was recorded. The background fluorescence (buffer and ethanol) was subtracted from the experimental value. Then all the data obtained were corrected for inner filter effect as all compounds studied showed the absorbance in both the albumin excitation and emission wavelengths (not shown). The spectral data of all compounds studied are presented in Table 1. The excitation and emission wavelengths $(278 / 340 \mathrm{~nm})$ were chosen as to provide the best fluorescence of albumin. The wavelength of $278 \mathrm{~nm}$ excites both tryptophan and tyrosine, however, tyrosine fluorescence is observed at $305 \mathrm{~nm}$. The emission registered at $340 \mathrm{~nm}$ responds to tryptophan fluorescence.

The PAHs are hardly soluble in water and for the highest concentration we observed their precipitation. To establish the maximal concentration of PAHs which is soluble in PBS, additional measurements of PAHs fluorescence spectrum were performed. An increase in background fluorescence indicates that the precipitation process has started as the light is scattered on the precipitated particles. In this way the maximal PAHs quantities soluble in PBS were determined.

When albumin was titrated with anthracene no fluorescence decrease was observed (Fig. 1a), however, for higher (above $1 \mu \mathrm{M}$ ) anthracene concentrations the fluorescence signal increased. The lack of albumin fluorescence quenching can suggest that there are no interactions between albumin and anthracene. Nevertheless, models 8 and 9 were applied because of the fluorescent properties of anthracene. No good fitting was achieved which indicates an absence of interactions. Hence, anthracene is assumed as non-binding to albumin.

For anthraquinone a slight drop in fluorescence intensity and no increase despite the fluorescent properties of anthraquinone were observed (Fig. 1b). All four equations considering low and high affinity as well as fluorescence and lack of fluorescence of the compound were fitted but no good fitting was achieved. This can indicate that either no interaction occurs or the models used in the study do not describe the anthraquinone-albumin interaction.

Two oxy-anthracene derivates, 9-anthracenemethanol and 9-anthraldehyde, exhibit similarly low affinity towards albumin. The obtained dissociation constants are $13.040 \pm 1.187$ and $7.252 \pm 0.781 \mu \mathrm{M}$, respectively (Table 2). The albumin fluorescence was quenched by these compounds at concentrations higher than $0.5 \mu \mathrm{M}$ for 9-anthracenemethanol and $0.1 \mu \mathrm{M}$ for 9-anthraldehyde, as seen in Fig. 1c and Fig. 1d. For 9-anthraldehyde a distinct rise of fluo- 
Anthracene

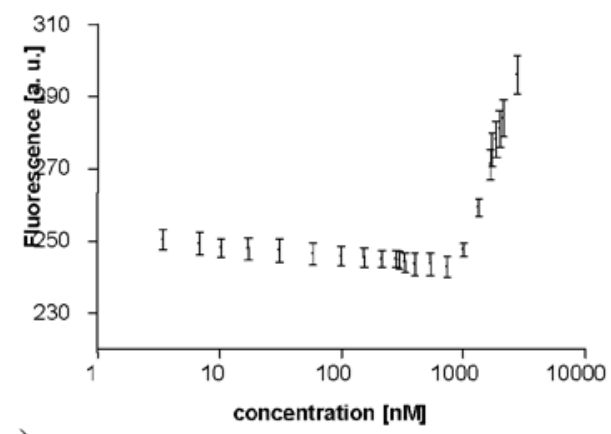

a)

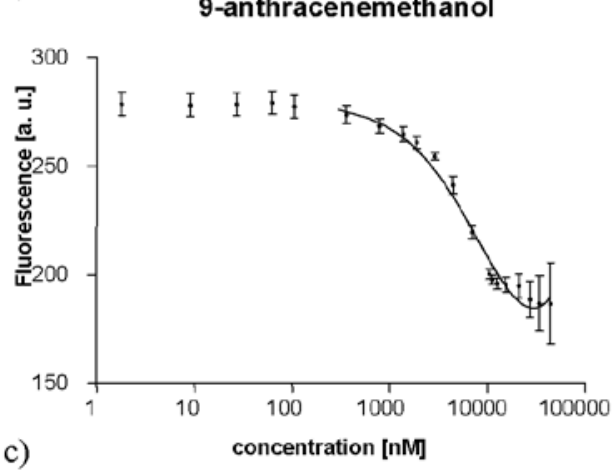

benzo[a]pyrene

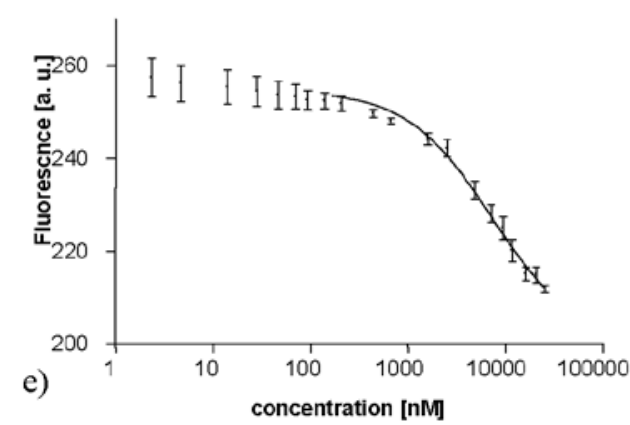

Antraquinone

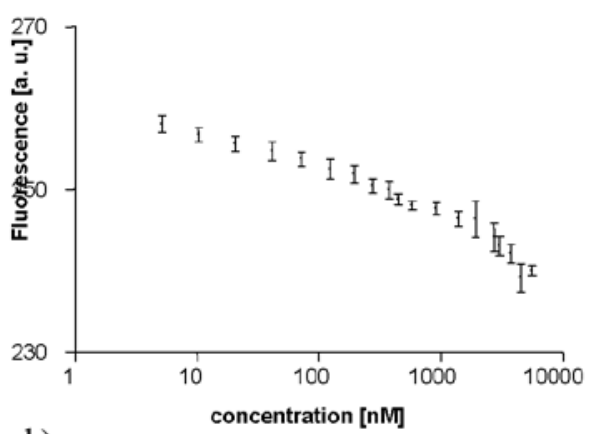

b)

9-anthraldehyde

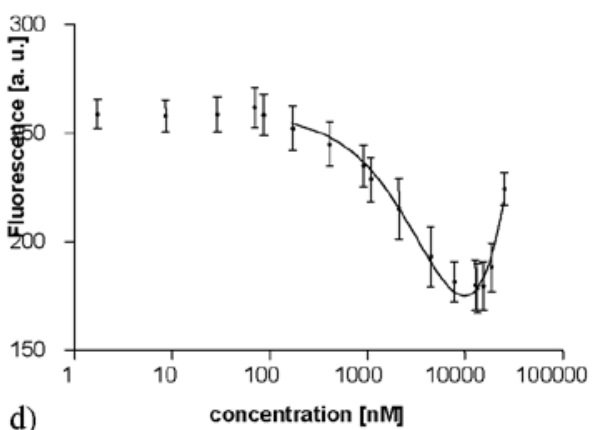

9-anthracenecarboxylic acid

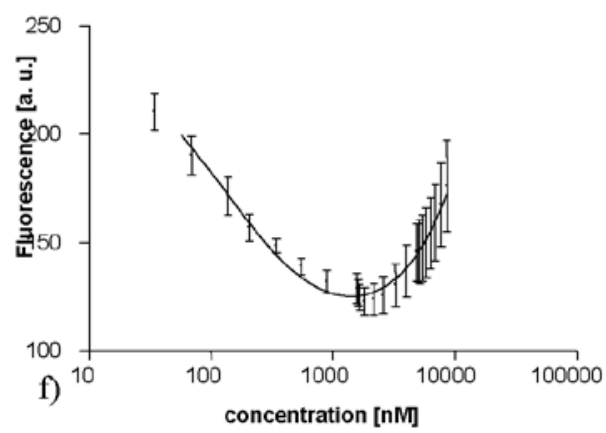

Figure 1. Albumin fluorescence when titrated with:

a, anthracene; b, anthraquinone; c, 9-anthracenemethanol; d, 9-anthraldehyde; e, benzo[a]pyrene; $\mathrm{f}$, 9-anthracenecarboxylic acid. Albumin at $0.5 \mu \mathrm{M}$ was titrated at $25^{\circ} \mathrm{C}$ in $0.05 \mathrm{M}$ PBS, pH 7.4. Albumin was excited at $278 \mathrm{~nm}$ and emission was detected at $340 \mathrm{~nm}$. Each experimental point is a mean of 5 titrations. The solid line is a best-fit theoretical curve.

rescence was observed for the concentrations above $10 \mu \mathrm{M}$, hence models assuming fluorescence of the compound were used. Model 9, assuming low affinity for albumin, was applied as it yielded a better value of $\mathrm{R}^{2}=0.92, P>0.9$. For 9-anthracenemethanol no distinct rise of fluorescence was observed, nevertheless fitting of various equations to experimental data yielded the best fit for the model assuming ligand fluorescence and a low affinity binding to albumin $\left(\mathrm{R}^{2}=0.95, P>0.9\right)$. The lower goodness of fit obtained for 9-antrhaldehyde can arise from the fact that the experimental points which are a mean of several measured values have relatively large standard deviations (Fig. 1d). Because a model assuming low-affinity interactions was applied, no data on stoichiometry was obtained.
When albumin was titrated with benzo[a]pyrene a weak fluorescence decrease was observed for concentrations higher than $0.1 \mu \mathrm{M}$. No fluorescence increase was observed even for the highest benzo[a]pyrene concentration (Fig. 1e). Since benzo[a]pyrene fluorescence was several times weaker than that of the other PAHs studied, equation 7, assuming no fluorescence of the compound, was used to calculate the dissociation constant. The curve fitting was good yielding the $\mathrm{R}^{2}-0.98$ and the dissociation constant was calculated to be $7.202 \pm 0.332 \mu \mathrm{M}$ (Table 2). This value indicates that benzo[a]pyrene binds to albumin with low affinity.

When albumin was titrated with dihydroxyanthraquinones fluorescence quenching was observed for all concentrations of 1,8-, 1,5- and 2,6-dihydroxy- 
Table 1. Literature and experimental spectral properties of PAHs

\begin{tabular}{|c|c|c|c|c|}
\hline PAH & $\begin{array}{l}\text { UV-VIS } \\
\lambda_{\max }\left(\varepsilon\left[\mathrm{M}^{-1} \mathrm{~cm}^{-1}\right]\right)\end{array}$ & $\begin{array}{l}\mathrm{Fl} \\
\lambda_{\max }\end{array}$ & $\begin{array}{l}\text { UV-VIS exp } \\
\lambda_{\max }\end{array}$ & $\begin{array}{l}\text { Fl exp } \\
\lambda_{\max }\end{array}$ \\
\hline Anthracene & $\begin{array}{l}252(220000)^{1} \\
323(2800)^{1} \\
339(5500)^{1} \\
356(8500)^{1} \\
374(8500)^{1}\end{array}$ & $\begin{array}{l}378^{2} \\
399^{2} \\
423^{2} \\
449^{2}\end{array}$ & $\begin{array}{l}258 \\
327 \\
343 \\
361 \\
380\end{array}$ & $\begin{array}{l}389 \\
410 \\
433\end{array}$ \\
\hline Anthraquinone & $\begin{array}{l}251(54000)^{1} \\
321(4800)^{1}\end{array}$ & - & $\begin{array}{l}257 \\
326\end{array}$ & - \\
\hline 9-Anthracenemethanol & $\begin{array}{l}256(14664)^{3} \\
332(505)^{3} \\
348(1125)^{3} \\
366(1767)^{3} \\
386(1577)^{3}\end{array}$ & $450(0.04)^{3}$ & $\begin{array}{l}259 \\
333 \\
349 \\
368 \\
388\end{array}$ & $\begin{array}{l}420 \\
398 \\
444\end{array}$ \\
\hline 9-Anthraldehyde & - & $463^{7}$ & $\begin{array}{l}264 \\
372 \\
403\end{array}$ & 499 \\
\hline Benzo[a]pyrene & $\begin{array}{l}297(63000)^{1} \\
347(12200)^{1} \\
365(25000)^{1} \\
385(29500)^{1}\end{array}$ & $\begin{array}{l}403^{2} \\
408.5^{2} \\
427^{2}\end{array}$ & $\begin{array}{l}299 \\
351 \\
369 \\
389\end{array}$ & $\begin{array}{l}412 \\
436 \\
463\end{array}$ \\
\hline 1,4-Dihydroxyanthraquinone & $\begin{array}{l}248(33000)^{1} \\
279(10800)^{1} \\
479(8200)^{1}\end{array}$ & $526^{4}$ & $\begin{array}{l}255 \\
475\end{array}$ & 550 \\
\hline 2,6-Dihydroxyanthraquinone & - & - & $\begin{array}{l}275 \\
303 \\
351\end{array}$ & 532 \\
\hline 1,8-Dihydroxyanthraquinone & $\begin{array}{l}430(2200)^{5} \\
284(2136)^{5} \\
272(2092)^{5} \\
253(1720)^{5}\end{array}$ & $586^{4}$ & $\begin{array}{l}259 \\
428\end{array}$ & 582 \\
\hline 1,5-Dihydroxyanthraquinone & $\begin{array}{l}436(-)^{4} \\
414(-)^{4}\end{array}$ & $582^{4}$ & $\begin{array}{l}259 \\
420\end{array}$ & 583 \\
\hline 9-Anthracenecarboxylic acid & $\begin{array}{l}330(28840)^{6} \\
346(58884)^{6} \\
363(69183)^{6} \\
383(27542)^{6}\end{array}$ & $470^{6}$ & $\begin{array}{l}259 \\
348 \\
365 \\
385\end{array}$ & 468 \\
\hline
\end{tabular}

${ }^{1}$ Atlas of Organic Compounds, Butterworths, Verlag Chemie, 1974; ${ }^{2}$ Dabestani \& Ivanov, (1999); ${ }^{3}$ Toulokhonova (2003); ${ }^{4}$ Morzocchi et al. (1998); ${ }^{5}$ Absorption Spectra in the Ultraviolet and Visible Region, Lang L. ed, Publishing House Hungarian Academy of Science, Budapest, Hungary (1963); ${ }^{6}$ Abdel-Mottaleb et al. (2000); ${ }^{7}$ Lin et al. (2000).

anthraquinones and for concentrations higher than $0.025 \mu \mathrm{M}$ of 1,4-dihydroxyanthraquinone (Figs 2a, b, c, d). All dihydroxyanthraquinones exhibit fluorescence when excited at $278 \mathrm{~nm}$, therefore models assuming ligand fluorescence were used in order to calculate dissociation constants. Indeed, for the highest PAHs concentrations ( $>5 \mu \mathrm{M}$ for 1,8-dihydroxyanthraquinone, $>3 \mu \mathrm{M}$ for 1,4-dihydroxyantaquinone and $>2.5 \mu \mathrm{M}$ for 2,6-dihydroxyanthraqui- none) a PAH-albumin solution fluorescence increase is observed with the exception of 1,5-dihydroxyanthraquinone.

The lack of fluorescence increase is probably caused by the fact that the concentration of 1,5-dihydroxyanthraquinone did not achieve $2 \mu \mathrm{M}$ and was too low to increase the fluorescence of solution. However, due to the spectral properties of this compound, models 8 and 9 were applied. For 
Table 2. Association constants and stoichiometry for albumin-PAHs complexes

\begin{tabular}{llll}
\hline PAH & $K_{\text {ass }}\left[\mu \mathrm{M}^{-1}\right]$ & $\mathrm{n}$ & Model \\
\hline Anthracene & - & - & 8,9 \\
Anthraquinone & - & - & $6,7,8,9$ \\
9-Anthracenemethanol & $0.077 \pm 0.007$ & - & 9 \\
9-Anthraldehyde & $0.138 \pm 0.015$ & - & 9 \\
Benzo[a]pyrene & $0.139 \pm 0.006$ & - & 7 \\
1,4-Dihydroxyanthraquinone & $0.347 \pm 0.021$ & - & 9 \\
2,6-Dihydroxyanthraquinone & $0.372 \pm 0.045$ & - & 9 \\
1,8-Dihydroxyanthraquinone & $0.526 \pm 0.034$ & 1.17 & 8 \\
1,5-Dihydroxyanthraquinone & $2.353 \pm 0.161$ & 1.08 & 8 \\
9-Anthracenecarboxylic acid & $7.519 \pm 0.678$ & 0.03 & 8
\end{tabular}

all dihydroxyanthraquinones two models were fitted ( 8 and 9) and better fit was chosen. For 1,4- and 2,6-dihydroxyanthraquinones the equations describing low-affinity interactions gave better results of fitting, whereas for the 1,8- and 1,5-dihydroxyanthraquinones Eqn. 8, describing high-affinity interactions, was more appropriate. The equations describe well the experimental points $\left(R^{2}>0.97\right)$ for three dihdroxyanthraquinones except for 2,6-dihydroxyanthraquinone $\left(\mathrm{R}^{2}=0.9\right)$. This lower value of $\mathrm{R}^{2}$ indicates that the goodness of fit is lower than for the other dihydroxyanthraquinones. Similarly to 9-antraldehyde this is possibly caused by the relatively large standard deviations of the experimental data obtained for 2,6-dihydroxyantraquinine. The obtained values of dissociation constants are: 2.883
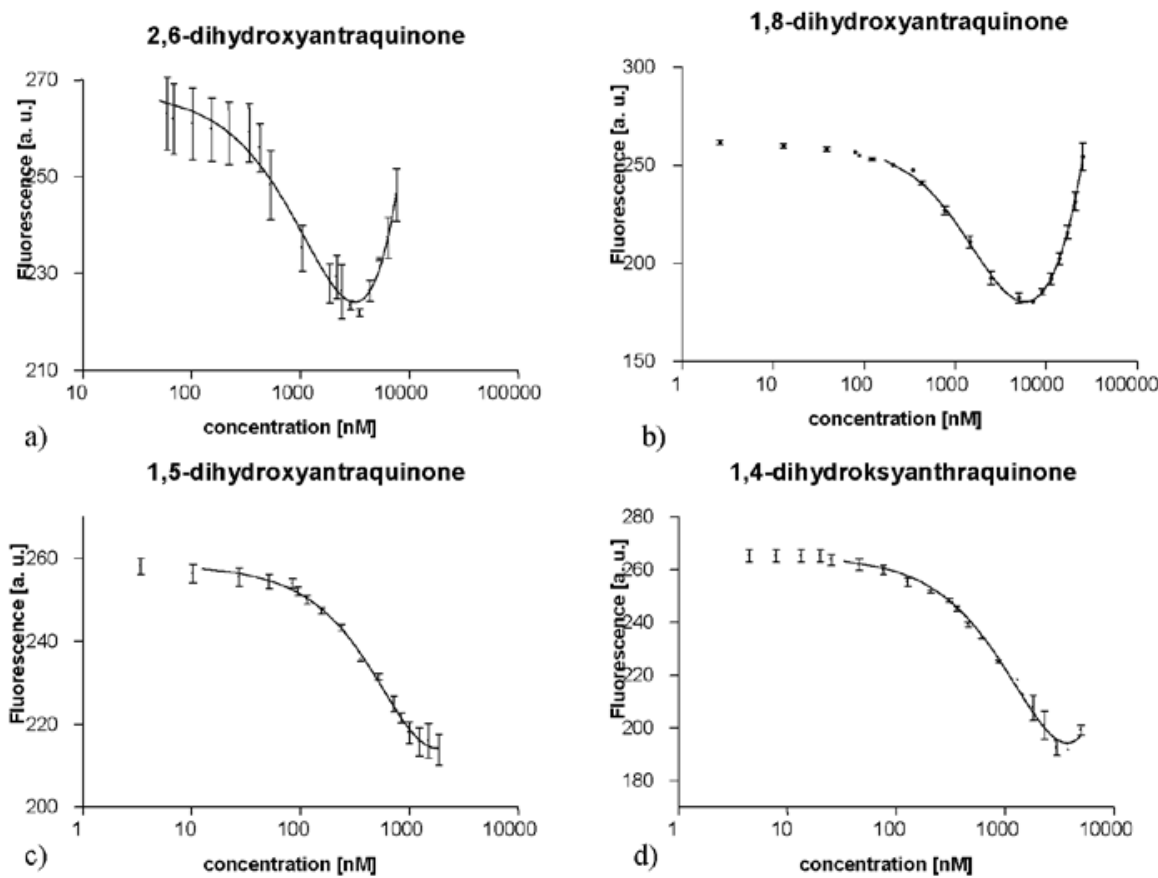

Figure 2. Titration curves for:

a, 2,6-Dihydroxyanthraquinone; b, 1,8-dihydroxyanthraquinone; c, 1,5-dihydroxyanthraquinone; d, 1,4-dihydroxyanthraquinone. Albumin, $0.5 \mu \mathrm{M}$, was titrated at $25^{\circ} \mathrm{C}$ in $0.05 \mathrm{M} \mathrm{PBS}$ at $\mathrm{pH} 7.0$. Albumin was excited at $278 \mathrm{~nm}$ and emission was detected at $340 \mathrm{~nm}$. Each experimental point is a mean of 5 titrations. The solid line is a best-fit theoretical curve. 
$\pm 0.171 \mu \mathrm{M}$ for 1,4-dihydroxyanthraquinone, 2.688 $\pm 0.325 \mu \mathrm{M}$ for 2,6-dihydroxyanthraquinone, 1.902 $\pm 0.123 \mu \mathrm{M}$ for 1,8-dihydroxyanthraquinone and $0.425 \pm 0.029 \mu \mathrm{M}$ for 1,5-dihydroxyanthraquinone (Table 2). For 1,8-dihydroxyantraquinone and 1,5dihydroxyanthraquinone model 7 was applied and the stoichiometry of binding was calculated: $n=1.17$ and $\mathrm{n}=1.08$, respectively.

9-Anthracenecarboxylic acid is the compound of the highest affinity toward albumin. Titration of albumin with this compound yielded a significant and rapid drop of albumin fluorescence for all concentrations used (Fig. 1f). Subsequent fluorescence rise for the highest 9-anthracenecarboxylic acid concentrations $>2 \mu \mathrm{M}$ was observed. Hence, models assuming ligand fluorescence were applied. The fitting of model 8 to experimental data with $\mathrm{R}^{2}=0.7$ yielded the dissociation constant $0.133 \pm 0.012 \mu \mathrm{M}$ and $\mathrm{n}=0.03$ (Table 2).

For all PAHs, the dissociation constants are collected in Table 2. The models used to calculate the constants and stoichiometry are also given there.

\section{DISCUSSION}

The binding affinities of 10 PAHs (structures presented in Fig. 3) for albumin were determined in this study in order to investigate the influence of the type and position of oxy-substituents in the PAH molecule. The method applied was albumin titration with the titrants containing increasing concentrations of the compound studied. Theoretical models assuming different types of interactions and different spectral properties of the compound were applied. Additionally, corrections for inner filter effect and albumin dilution were introduced to obtain accurate values of dissociation constants.

Up to now, the binding constants to albumin have been established for drugs or endogenous products of metabolism (e.g. bilirubin, free fatty acids) as it is an important aspect of the activity of these substances. There is no literature data available on PAHs and other pollutants' affinity toward albumin.

Several methods have been used to determine the ligand-protein binding constants, fluorescence quenching being one of them.
The association constant describing the ligand-protein bond strength was commonly calculated from curve fitting to experimental points. Some researchers fit the equation described by Scatchard to experimentally obtained values of albumin fluorescence and from this determine the affinity constant and capacity (Levine, 1977; Guo et al., 2004). Calculation of the constants from the Scatchard equation was also a method used by Paal et al. (2001), however, the fluorescence intensity was not measured, but the concentrations of unbound ligand which were determined from RP-HPLC measurements.

In our work the quenching of albumin fluorescence due to increasing concentrations of ligand in the titrant, was measured and equations describing the fluorescence dependence upon concentration of the ligand were applied.

Epps et al. $(1995 ; 1999)$ used this method with success to determine the affinity of drugs to albumin. This method was also applied by Niedzwiecka et al. (2002) and Zuberek et al. (2003) to determine the interaction between the cap-binding protein eIF4E with mRNA 5' end and its analogs. This method allows us to calculate the dissociation constants of the studied compound-albumin complexes.

Since our calculation gave dissociation constants and literature data present mostly association ones, we recalculated the dissociation constants to the association ones to make comparison of our results easier. They are shown in Table 2. The values of the dissociation constants were in the range 13.040-0.111 $10^{-6} \mathrm{M}$, which gives the association constants in the range 0.077-9.009 $10^{6} \mathrm{M}^{-1}$.

The association constants obtained by us indicate that the affinity of PAHs toward albumin is similar to the affinity of drugs and endogenous substances, e.g. the highest binding constants were observed for bilirubin and long-chain fatty acids $\left(K_{\text {ass }}\right.$ $\approx 10^{7}-10^{8} \mathrm{M}^{-1}$ ) (Levine, 1977; Paal et al., 2001). The association constants obtained for Furosemid was 13 $\times 10^{6} \mathrm{M}^{-1}$ (Levine, 1977), for Warfarin $0.29 \times 10^{6} \mathrm{M}^{-1}$ and for Dansylsulfonamide $0.13 \times 10^{6} \mathrm{M}^{-1}$ (Epps et al., 1995).

Epps et al. (1995) determined also the constants for other nonfluorescent drugs by the method of displacement of fluorescent probe, such as Ibuprofen which the constant $0.25 \times 10^{6} \mathrm{M}^{-1}$ and for a low-affinity drug - a nonpeptidic HIV protease inhibitor U-105,665 it is $0.018 \times 10^{6} \mathrm{M}^{-1}$.

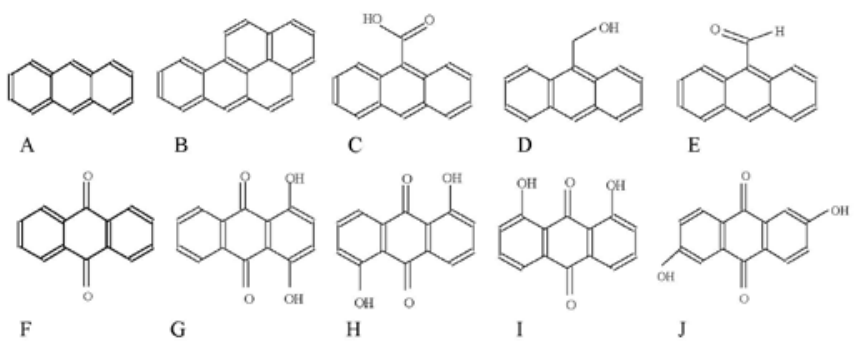

$\mathrm{H}$
Figure 3. Structures of PAHs:

$\mathrm{A}$, anthracene; B, benzo[a]pyrene; C, 9-anthracenecarboxylic acid; D, 9-anthracenemethanol; E, 9-anthraldehyde; F, anthraquinone; G, 1,4dihydroxyanthraquinone; $\mathrm{H}, \quad$ 1,5-dihydroxyanthraquinone; I, 1,8-dihydroxyanthraquinone; J, 2,6-dihydroxyanthraquinone 
In a recent paper Epps et al. (1999) studied the association constants and found the following values: for high affinity drugs: 0.45 and $2.08 \times 10^{6}$ $\mathrm{M}^{-1}$ and for low-affinity $0.054 \times 10^{6} \mathrm{M}^{-1}$. The studies were also conducted with bovine serum albumin and the following values of the constants were obtained for hemin - $50 \times 10^{6} \mathrm{M}^{-1}$ (Silva et al., 2004) and for the antibiotic Gatifloxacin $0.1 \times 10^{6} \mathrm{M}^{-1}$ (Guo et al., 2004).

Paclitaxel, an antitumor drug, was shown to bind to albumin at one site with a high affinity of $2.4 \times 10^{6} \mathrm{M}^{-1}$ and at a second one with a moderate affinity of $0.1 \times 10^{6} \mathrm{M}^{-1}$ (Paal et al., 2001).

Among the PAHs studied, 9-antracenocarboxylic acid $\left(9.0 \times 10^{6} \mathrm{M}^{-1}\right)$ and all dihydroxyanthraquinones $\left(0.3-2.4 \times 10^{6} \mathrm{M}^{-1}\right)$ demonstrate a high affinity toward albumin. Benzo[a]pyrene and 9-antraldehyde $\left(0.14 \times 10^{6} \mathrm{M}^{-1}\right)$ are recognized to bind to albumin with moderate affinity and 9-anthracenemethanol $\left(0.08 \times 10^{6} \mathrm{M}^{-1}\right)$ with low affinity. These results indicate that some of PAHs derivates can bind as strongly as drugs and endogenous substances often very important for organism functioning.

Anthracene and anthraquinone were determined in this study as non-binding to albumin. The presence of aldehyde or methyl substituents only slightly increases the affinity of anthracene derivatives for albumin.

A very interesting result was obtained for benzo[a]pyrene. It is believed that PAHs must be metabolised to oxy-derivatives which in this form only are able to interact with biological molecules. We obtained good fitting of a theoretical curve calculated for weak ligand affinity for experimental points. This suggests that the quenching of albumin fluorescence is due to weak albumin-benzo[a]pyrene interaction.

Dihydroxyanthraquinones exhibit relatively high values of affinity constants. It is probably the result of albumin interaction with these PAHs through hydrophobic stacking and $\mathrm{H}$-bonds involving $=\mathrm{O}$ and $-\mathrm{OH}$ groups (Fabriciova et al., 2004).

The highest affinity was determined for anthracenecarboxylic acid $(7.519 \pm 0.678 \mu \mathrm{M})$ which indicates that carboxyl group is the most reactive toward albumin. This result is in agreement with previous findings that fatty acids and bilirubin (Levine, 1977) or Ibuprofen (Epps et al., 1995) exhibit very high affinity toward albumin. All these molecules contain - $\mathrm{COOH}$ groups, however, the structure of these compounds is complex and it is difficult to decide if the high affinity toward albumin is caused only by the presence of the $-\mathrm{COOH}$ groups.

The studied compounds can be divided into two groups. The first: anthracene substituted with groups containing carbon on gradually increasing oxidated state: carbohydroxyl $(\mathrm{C}-\mathrm{OH})$, carbonyl
$(\mathrm{C}=\mathrm{O})$ and carboxylic $(\mathrm{COOH})$; this means anthracenemethanol, aldehyde and acid ones. In this case the strength of PAHs affinity toward albumin increases together with the carbon oxidated state.

The second group are anthracene derivatives containing both hydroxyl and carbonyl groups attached in various position in the molecule - dihydroxyanthraquinones. The addition of two carbonyl groups $(\mathrm{C}=\mathrm{O})$ to the anthracene molecule (anthraquinone) does dot increase the binding affinity for albumin; only the presence of both carbonyl and hydroxyl groups distinctly increases the affinity. The highest albumin affinity was shown by 1,5-dihydroxyanthraquinone, then by 1,8 - and the least affinity was shown by 2,6- and 1,4-dihydroxyanthraquinone. Up to 5-fold differences in affinity constants were observed, which indicates that the position of the hydroxyl substituents in the anthracene molecules determines its binding affinity.

High affinity constants were achieved when both hydroxyl and carbonyl groups were located next to each other. The strongest effect is when hydroxyl groups are located on two opposite sides of the molecule (in positions 1 and 5). The configuration of the $=\mathrm{O}$ and $-\mathrm{OH}$ groups in 1,5-dihydroxyanthraquinone is very similar to the $-\mathrm{COOH}$ group in anthracenecarboxylic acid. Moreover, the presence of $=\mathrm{O}$ and $-\mathrm{OH}$ substituents on the opposite sides of the anthracene skeleton gives more possibilities to interact with albumin as this molecule can bind at either side.

The presence of two $-\mathrm{OH}$ groups close to the $=\mathrm{O}$ group (1,8-dihydroxyanthraquinone) also is beneficial, however, the binding strength is lower due to a steric barrier caused by the close proximity of the $=\mathrm{O}$ group in position 9 and the $-\mathrm{OH}$ groups in positions 1 and 8 .

This observation shows that the affinity toward biological molecules increases with the increase in the electrophilic properties of PAH molecule caused by hydroxyl groups. Hence, PAH metabolic transformation seems to be a crucial step in the PAH distribution into tissues and cells.

All compounds studied by us can be a model of oxygen-containing PAHs obtained after the metabolism of parent polyaromatic compounds and also after their subsequently deeper oxidation in living organism. They can be also examples of products obtained by polyaromatics combustion and hence could be present in, e.g., cigarette smoke.

Similarly like drugs and endogenous substances, PAHs bound to albumin can be distributed all over the body; hence PAHs can reach the organs which are not directly exposed to PAHs. Then they can disturb this organ's metabolism, eventually leading to the carcinogenesis. 
On the other hand, as it is described for drugs, binding of ligands with albumin can decrease their (here PAHs) concentration in the blood, hence it can reduce the harmful effect of exposure to PAHs. With respect to this, the albumin capacity could be a very important factor leading to scavenging of PAHs biological effect on humans exposed to this pollutant. If a single molecule of albumin is able to bind more than one PAH molecule (as it has been shown in the literature) it can reduce the level of pollutants and its metabolites in the blood even more effectively.

We were able to determine the binding stoichiometry of three compounds, which showed high affinity toward albumin: 1,5-dihydroxyanthraquinone $(\mathrm{n}=1.08), 1,8$-dihydroxyanthraquinone $(\mathrm{n}=$ 1.17) and 9-anthracenecarboxylic acid $(\mathrm{n}=0.03)$. The results obtained for dihydroxyanthraquinones suggest that only one $\mathrm{PAH}$ molecule can bind to one molecule of albumin, which agrees with previous findings of Tannenbaum and Brunmark (Tannenbaum et al., 1993; Brunmark et al., 1997) suggesting that albumin has one specific binding site for PAHs - either histidine 146 or lysine 137.

The stoichiometry of binding of anthracenecarboxylic acid to albumin $(\mathrm{n}=0.03)$ found by us reveals low capacity of HSA. This indicates that although anthracenecarboxylic acid binds to albumin the most strong of all PAHs studied, a relatively small number of molecules interact with albumin. However, this can be explained by the fact that the carboxylic group of anthracenecarboxylic acid is likely to be dissociated at $\mathrm{pH}$ 7.4. Hence, some anthracenecarboxylic acid molecules, a relatively weak acid exist in anionic form. These molecules are able to form strong ionic bonds with albumin. The molecules which are not dissociated are able to interact with albumin through the much weaker hydrogen bonds. Thus only a fraction of the anthracenecarboxylic acid molecules introduced to albumin solution participate in strong albumin binding.

Another aspect of albumin-PAHs interaction is the site of binding. Albumin is a protein which plays a significant role in transporting many substances and has many binding sites.

Up to now only few studies have been performed to determine albumin binding sites for PAHs. Binding of PAHs diolepoxides was studied and histidine-146, lysine-137 (subdomain IB) and lysine-385 (subdomain IIIA) were proposed as the binding sites.

Fabriciova et al. (2004), studied binding of two dihydroxyanthraquinones: 1,8-dihydroxyanthraquinone and 1,4-dihydroxyanthraquinone to human serum albumin. They proposed albumin subdomains IIIA and IIA to be the dihydroxyanthraquinone binding region. However, in the presence of fatty acids, in the case of fraction $\mathrm{V}$ albumin, only sub- domain IIA is the binding region for these PAHs because subdomain IIIA is a specific primary binding site of fatty acids.

The binding sites for the PAHs studied by us can be proposed also on the basis of the binding sites characteristic for drugs which contain aromatic rings and oxy-substituents in the structure. There are literature data on a number of such drugs, e.g.: Chloropromazine (three aromatic rings), Ibuprofen (one ring, $-\mathrm{COOH}$ ), Oxazepan (two rings, $=\mathrm{O},-\mathrm{OH}$ ), Warfarin (three rings, $=\mathrm{O},-\mathrm{OH}$ ), Tolmetin (one ring, $-\mathrm{COOH}$ ) as well as Ochratoxin (two rings $-\mathrm{OH},=\mathrm{O}$ ) and its derivatives, hydroquinone and on O-methylated derivative. All these drugs were shown to bind either in subdomain IIA or IIIA (Epps et al, 1995; Qiu et al., 1998; Perry et al., 2003; Silva et al., 2004).

Thus, while summarizing the above information, we can conclude that albumin subdomains IB, IIA or IIIA are specific for the PAHs studied. However, in the presence of fatty acids in albumin fraction V only IB and IIA should be considered as binding regions for PAHs.

The albumin fluorescence quenching by PAHs studied can indicate that the compounds interact with albumin in the surrounding of the albumin fluorescent residue tryptophan 214 localized in subdomain IIA. The binding of a PAH molecule to albumin can affect its fluorescence by acting as a tryptophan quencher via collisional or energy transfer. It is also possible that the $\mathrm{PAH}$ molecule physically interacts with the tryptophan, by which changes the polarity of its environment or prevents solvent accessibility, which complexation also influences the fluorescence. This could suggest also that subdomains IB and IIA are specific binding regions of anthracene derivatives.

However, fluorescence quenching can be also caused by binding of the ligand to a site distant from the tryptophan. Such interaction can change the protein conformation which can influence the tryptophan environment and in this way change the fluorescence intensity (Mayers, 2000).

For all compounds studied a fluorescence drop was observed - the most rapid one for anthracenecarboxylic acid, which can indicate that this compound binds to albumin very close to the tryptophan. We can suppose also that anthraquinone binds to albumin weakly and probably far from tryptophan 214 , since despite the observed fluorescence intensity quenching, we were not able to fit a theoretical equation to the experimental data. This indicates that this compound does not quench tryptophan fluorescence directly but, possibly by binding to a remote site, it changes the albumin conformation.

Although the results achieved by us are not decisive, they are in accordance with the literature 
data on drug or PAHs binding to albumin. They indicate that subdomains IB or IIA are the albumin specific region for anthracene derivatives binding.

In order to determine the exact binding site of the compounds studied displacement measurements should be conducted. Also simultaneous measurement of tyrosine and tryptophan fluorescence, extended also to fatty-acid-free albumin or even bovine albumin, could determine the binding sites. These investigations are in progress.

Summarizing, since anthracene was shown not to interact with albumin, its metabolic transformations to oxy-derivatives is an essential condition to produce such PAHs forms which are able to interact with biological molecules. Our results indicated that oxy-PAHs present in the environment can immediately create adducts with albumin and the affinity of these oxy-derivatives for albumin, depends considerably on the type and position of substituents in the PAH molecule.

\section{REFERENCES}

Abdel-Mottaleb MSA, Galal HR, Dessouky AFM, El-Naggar M, Mekkawi D, Ali SS, Attya GM (2000) Fluorescence and photostability studies of anthracene-9-carboxylic acid in different media. Int $J$ Photoenergy 2: 47-53.

Boysen G, Hecht SS (2003) Analysis of DNA and protein adducts of benzo[a]pyrene in human tissues using structure-specific methods. Mutat Res 543: 17-30.

Brunmark P, Harriman S, Skipper PL, Wishnok JS, Amin S, Tannenbaum SR (1997) Identification of subdomain IB in human serum albumin as a major binding site for polycyclic aromatic hydrocarbons epoxides. Chem Res Toxicol 10: 880-886.

Dabestani R, Ivanov N (1999) A compilation, spectroscopic and photophysical properties of polycyclic aromatic hydrocarbons. Photochem Photobiol 70: 10-34.

Ehrenberg L, Granath F, Tornqvist M (1996) Macromolecule adducts as biomarkers of exposure to environmental mutagens in human populations. Environ Health Perspect 104: 423-428.

Epps DE, Raub TJ, Kezdy FJ (1995) A general, wide-range spectrofluorimetric method for measuring the site-specific affinities of drugs toward human serum albumin. Anal Biochem 227: 342-350.

Epps DE, Raub TJ, Caiolfa V, Chiari A, Zamai M (1999) Determination of the affinity of drugs toward serum albumin by measurement of the quenching of the intrinsic Tryptophan fluorescence of the protein. J Pharm Pharmacol 51: 41- 48.

Fabriciova G, Sanchez-Cortes S, Garcia-Ramos JV, Miskovsky P (2004) Joint application of micro-Raman and surface-enchanced Raman spectroscopy to the interaction study of the antitumoral anthraquinone drugs danthron and quinizarin with albumins. J Raman Spectrosc 35: 384-389.

Garner RC (1998) The role of DNA adducts in chemical carcinogenesis. Mut Res 402: 67-75.

Guo M, Zou J-W, Yi P-G, Shang Z-C, Hu G-X, Yu Q-S (2004) Binding interaction of gatifloxacin with bovine serum albumin. Anal Sci 20: 465-470.
Hecht SS, Chen M, Yagi H, Jerina DM, Carmella SG (2003) $\mathrm{r}-1, \mathrm{t}-2,3, \mathrm{c}-4$-Tetrahydroxy-1,2,3,4-tetrahydrophenanthrene in human urine: a potential biomarker for assessing polycyclic aromatic hydrocarbon metabolic activation. Cancer Epidemiol Biomark Prev 12: 1501-1508.

Honey S, O'Keefe P, Drahushuk AT, Olson JR, Kumar S, Sikka HC (2000) Metabolism of benzo(a)pyrene by duck liver microsomes. Comp Biochem Physiol C 126: 285-292.

Jacob J, Seidel A (2002) Biomonitoring of polycyclic aromatic hydrocarbons in human urine. J Chromatogr $B$ 778: 31-47.

Kragh-Hansen U (1990) Structure and ligand binding properties of human serum albumin. Dan Med Bull 37: 57-84.

Kumar S, Chang RL, Wood AW, Xie JG, Huang MT, Cui XX, Kole PL, Sikka HC, Balani SK, Conney AH, Jerina DM (2001) Tumorigenicity of racemic and optically pure bay region diol epoxides and other derivatives of the nitrogen heterocycle dibenz[a,h]acridine on mouse skin. Carcinogenesis 22: 951-955.

Lecoq S, Chalvet O, Strapelias H, Grover PL, Philips DH, Duquesne M (1991) Microsomal metabolism of dibenz[a,c]anthracene, dibenz[a,h]anthracene and dibenz[a,j]anthracene to bisdihydrodiols and polyhydroxylated products. Chem Biol Interact 80: 261-279.

Lee BM, Baoyun Y, Herbert R, Hemminki K, Perera FP, Santella RM (1991) Immunologic measurement of polycyclic aromatic hydrocarbon-albumin adducts in foundry workers and roofers. Scand J Work Environ Health 17: 190-194.

Lee W, Shin HS, Hong JE, Pyo H, Kim Y (2003) Studies on the analysis of benzo(a)pyrene and its metabolites in biological samples by using high performance liquid chromatography/fluorescence detection and gas chromatography/mass spectrometry. Bull Korean Chem Soc 24: 559-565.

Levine RL (1977) Fluorescence quenching studies of the binding of bilirubin to albumin. Clin Chem 23: 22922301.

Lin Z-P, Aue WA (2000) Gas-phase luminescence of aromatic carbonyl compounds in excited nitrogen at atmospheric pressure. Can J Chem 78: 95-117.

Ma JK, Fu PP, Luzzi LA (1977) Protein binding of benz[a]anthracene and benzo[a]pyrene. J Pharm Sci 66: 209-213.

Mayers RA (2000) Ladokhin Encyclopedia of Analytical Chemistry, pp 5762-5779, John Wiley \& Sons LTD Chichester.

Morzocchi MP, Mantini AR, Casu M, Smulevich G (1998) Intramolecular hydrogen bonding and excited state proton transfer in hydroxyanthraquinones as studied by electronic spectra, resonance Raman scattering and transform analysis. J Chem Phys 108: 534-549.

Niedzwiecka A, Marcotrigiano J, Stepinski J, Jankowska-Anyszka M, Wyslouch-Cieszynska A, Dadlez M, Gingras A-C, Mak P, Darzynkiewicz E, Sonenberg N, Burley SK, Stolarski R (2002) Biophysical studies of eIF4E cap-binding protein: recognition of mRNA 5' cap structure and synthetic fragments of eIF4G and 4E-BP1 proteins. J Mol Biol 319: 615-635.

Nielsen PS, Okkels H, Sigsgaard T, Kyrtopoulos S, Autrup $\mathrm{H}$ (1996) Exposure to urban and rural air pollution: DNA and protein adducts and effect of glutathione-Stransferase genotype on adduct levels. Int Arch Environ Health 68: 170-176.

Otto S, Bhattacharyya KK, Jefcoate CR (1992) Polycyclic aromatic hydrocarbon metabolism in rat adrenal, ovary and testis microsomes is catalyzed by the same novel 
cytochrome P450 (P450RAP). Endocrinology 131: 30673076.

Paal K, Muller J, Hegedus L (2001) High affinity binding of paclitaxel to human serum albumin. Eur J Biochem 268: 2187-2191.

Pangrekar J, Kole PL, Honey SA, Kumar S, Sikka HC (2003) Metabolism of chrysene by brown bullhead liver microsomes. Toxicol Sci 71: 67-73.

Pashko LL, Schwartz AG (1996) Inhibition of 7,12-dimethylbenz[a]anthracene-induced lung tumorigenesis in A/J mice by food restriction is reversed by adrenalectomy. Carcinogenesis 17: 209-212.

Perry JL, Il'ichev YV, Kempf VR, McClendon J, Park G, Manderville RA, Rüker F, Dockal M, Simon JD (2003) Binding of ochratoxin a derivatives to human serum albumin. J Phys Chem B 107: 6644-6647.

Philips DH (1999) Polycyclic aromatic hydrocarbons in the diet. Mutat Res 443: 139-247.

Prodi G, Grilli S, Mazzullo M, Colacci A, Arfellini G (1984) Comparison between photo-induction and microsomal activation of polycyclic hydrocarbons with different oncogenic potency. Toxicol Pathol 12: 185-188.

Qiu Y, Burlingame AL, Benet LZ (1998) Mechanisms for covalent binding of benoxaprofen glucuronide to human serum albumin. Drug Metabol Disp 26: 246-256.

Quiming NS, Vergel RB, Nicolas MG, Villanueva JA (2005) Interaction of bovine serum albumin and metallothionein. J Health Sci 51: 8-15.

Ruchirawat M, Mahidol Ch, Tangjarukij Ch, Pui-ock S, Jensen O, Kampeerawipakorn O, Tuntaviroon J, Aramphongphan A, Autrup H (2002) Exposure to genotoxins present in ambient air in Bangkok, Thailand - particle associated polycyclic aromatic hydrocarbons and biomarkers. Sci Tot Env 287: 121-132.

Santella RM, Perera FP, Young TL, Zhang Y-J, Chiamprasert S, Tang D, Wang LW, Beachman A, Lin J-H,
DeLeo VA (1995) Polycyclic aromatic hydrocarbonDNA and protein adducts in coal tar treated patients and controls and their relationship to glutathione Stransferase genotype. Mutat Res 334: 117-124.

Silva D, Cortez CM, Louro SRW (2004) Quenching of the intrinsic fluorescence of bovine serum albumin by chlorpromazine and hemin. Braz J Med Biol Res 37: 963-968.

Sram RJ, Binkova B (2000) Molecular epidemiology studies on occupational and environmental exposure to mutagens and carcinogens, 1997-1999. Environ Health Perspect 108: 57-70.

Tannenbaum SR, Skipper PL, Wishnok JS, Stillwell WG, Day BW, Taghizadeh K (1993) Characterization of various classes of protein adducts. Environ Health Perspect 99: 51-5.

Toulokhonova I, Bjerke-Kroll B, West R (2003) Synthesis and photophysical properties of novel fluorescent silicones. J Organ Chem 686: 101-104.

Wang Ch-J, Lee M-J, Chang M-Ch, Lin J-K (1995) Inhibition of tumor promotion in benzo[a]pyrene-initiated CD-1 mouse skin by crocetin. Carcinogenesis 16: 187-191.

Willett KL, Gardinali PR, Lienesch LA, Di Giulio RT (2000) Comparative metabolism and excretion of benzo(a)pyrene in 2 species of Ictalurid catfish. Toxicol Sci 58: 68-76.

Zuberek J, Wyslouch-Cieszynska A, Niedzwiecka A, Dadlez M, Stepinski J, Augustyniak W, Gingras A-C, Zhang Z, Burley SK, Sonenberg N, Stolarski R, Darzynkiewicz E (2003) Phosphorylation of eIF4E attenuates its interaction with mRNA $5^{\prime}$ cap analogs by electrostatic repulsion: Intein-mediated protein ligation strategy to obtain phosphorylated protein. RNA 9: 52-61. 